\title{
Intratumoral injection of cisplatin in various concentrations of ethanol for cisplatin-resistant lung tumors
}

\author{
QI NIU ${ }^{1}$, WEI WANG ${ }^{1},{\text { QIAN } L^{2}}^{2}$, YONG LI ${ }^{1}$, DOUGLAS M. RUDEN ${ }^{3}$ and FENGHUA WANG ${ }^{4}$ \\ ${ }^{1}$ Department of Medical Oncology, 309 PLA Hospital, Haidian, Beijing 100091; ${ }^{2}$ Department of Internal Medicine, \\ Beijing Language and Culture University Hospital, Haidian, Beijing 100083, P.R. China; \\ ${ }^{3}$ Department of Obstetrics and Gynecology, Institute of Environmental Health Sciences, \\ C.S. Mott Center for Human Health and Development, Wayne State University, \\ Detroit, MI 48201, USA; ${ }^{4}$ Department of Pathology, 309 PLA Hospital, \\ Haidian, Beijing 100091, P.R. China
}

Received September 23,2013; Accepted April 4, 2014

DOI: $10.3892 / \mathrm{mco} .2014 .275$

\begin{abstract}
Cisplatin [cis-diammineplatinum dichloride (DDP)] resistance is a major limitation in the treatment of lung cancer. We previously demonstrated that DDP dissolved in 5\% ethanol (5\% ethanol-DDP) injected intratumorally was able to eradicate DDP-resistant lung tumors and prolong survival, as 5\% ethanol improved DDP delivery to the tumor. The present study aimed to investigate the efficacy of DDP in various concentrations of ethanol and determine the optimal ethanol concentration in which DDP exhibits optimal efficacy in reducing tumor volume and prolonging survival. The efficiency of DDP dissolved in 2, 5, 10, 20 and 50\% ethanol (v/v) in DDP-resistant A549/DDP lung tumor-bearing Balb/C nude mice was investigated. Tumor growth and survival were evaluated in all the treatment groups. Microvessel density in xenograft tumor tissues was measured by immunohistochemistry. Our results revealed that 5\% ethanol-DDP exhibited the highest efficiency in reducing tumor volume and prolonging survival among all the investigated ethanol-DDP combinations. We found that 5\% ethanol-DDP produced the most significant inhibition of tumor angiogenesis among all the remaining ethanol-DDP combinations, while treatment with ethanol alone increased tumor angiogenesis. In conclusion, 5\% ethanol-DDP produced the strongest tumor growth inhibition and longest survival among all the investigated ethanol-DDP combinations, possibly providing a novel therapeutic strategy for improving the survival of patients with DDP-resistant lung cancer. The potent inhibition of tumor angiogenesis by 5\% ethanol-DDP may be one of the mechanisms underlying its superior efficiency.
\end{abstract}

Correspondence to: Professor Qi Niu, Department of Medical Oncology, 309 PLA Hospital, A17 Heishanhu Road, Haidian, Beijing 100091, P.R. China

E-mail: qi_niu@hotmail.com

Key words: lung cancer, cisplatin, ethanol, cisplatin resistance

\section{Introduction}

Lung cancer, including small-cell lung cancer (SCLC) and non-small-cell lung cancer (NSCLC), is the most common type of cancer worldwide (1). Cisplatin [cis-diammineplatinum dichloride (DDP)]-based chemotherapy has long been used as first-line treatment for patients with SCLC and advanced NSCLC (2-3). However, DDP resistance and tumor relapse, which are likely mediated by cancer stem-like cells (CSCs) or side-population (SP) cells, invariably occur (4-6).

CSCs or SP cells express high levels of breast cancer resistance protein (ABCG2), which acts as an adenosine triphosphate-dependent membrane transporter. ABCG2 efficiently effluxes a variety of chemotherapeutic agents, such as DDP, in chemoresistant cancer cells and may be the main mechanism underlying resistance to chemotherapy (5,7-8).

Consistently, previous studies by Kim et al (9) and Tang et al (10) reported that decreased platinum accumulation in NSCLC tumor tissues and SCLC cells may be an important mechanism underlying platinum resistance in NSCLC and SCLC cells in the clinical setting.

Over the last 20 years, high concentrations of ethanol (>95\%) alone have been successfully used for the ablation of various types of tumors, particularly liver tumors sized $<3 \mathrm{~cm}$ (11-12). Although high concentrations of ethanol may destroy various types of smaller tumors through inducing tumor cell dehydration and necrosis, protein degeneration in larger tumors may result in boundary formation, which causes some areas to be left unharmed (11-12). Moreover, Tan et al (13) and Forsyth et al (14) demonstrated that the administration of ethanol alone may stimulate angiogenesis and promote tumor growth.

We previously demonstrated that a low concentration of ethanol (5\%) was able to potently inhibit the ABCG2 pump in lung CSCs (15). The inhibition of ABCG2 results in the deposition of cytotoxic DDP into lung CSCs and other cancer cells, efficiently eliminating them (15-16). DDP in 5\% ethanol (5\% ethanol-DDP) injected intratumorally was able to eradicate DDP-resistant lung tumors and extend survival by eliminating lung CSCs and non-CSC cancer cells in our previous xenograft study (15). 
However, there may be an optimal concentration of ethanol in which DDP exhibits its highest efficacy in reducing tumor volume and extending survival compared to $5 \%$ ethanol-DDP.

Therefore, the present study aimed to compare the efficiency of DDP in 2, 10, 20 and 50\% ethanol to that of 5\% ethanol-DDP for the treatment of DDP-resistant lung tumor-bearing nude mice. In addition, we investigated the potential mechanisms underlying the differences in DDP efficiency by immunohistochemistry (IHC) analysis of microvessel density (MVD) in xenograft tumor tissues.

\section{Materials and methods}

Ethics statement. All animal experiments were conducted with the approval of the Institutional Animal Care and Use Committee of the China Agricultural University (Haidian, China). The conditions of the animals were monitored daily for evidence of illness. Four steps were taken to minimize the suffering of the animals as follows: i) air exchange, temperature, humidity, noise, light intensity and light cycles were maintained within limits compatible with the health and well-being of the mice; ii) cleaning practices were monitored on a regular basis to ensure effective hygiene and sterile sanitation; iii) to avoid unnecessary harm, the agents were injected gently; and iv) mice showing severe distress, including infection, ulceration, cachexia, inability to ambulate and moribund, were humanely euthanized by cervical dislocation in accordance with animal care protocols. All the mice were euthanized after 180 days of observation. Two mice that exhibited severe distress due to biting infection in the tumors succumbed to non-tumor-related factors.

A number of mice in this study developed sizeable tumors, as survival research requires as long an observation time as possible. Moreover, we previously reported that mice receiving ethanol treatment developed larger tumors and better general condition compared to control mice. As a result, mice with sizeable tumors appeared to be in a good overall condition even up to 3 weeks prior to the onset of severe distress.

Cancer cell culture and reagents. The DDP-resistant A549/DDP human lung adenocarcinoma cell line was obtained from the American Type Culture Collection (Rockville, MD, USA) and was routinely cultured in RPMI medium supplemented with $10 \%$ fetal bovine serum (Invitrogen China Ltd., Beijing, China) and $2 \mu$ mol DDP (Qilu Pharmaceutical Co., Ltd., Jinan, China). The A549/DDP cells were cultured in complete RPMI medium without DDP for 3 days prior to use. Ethanol ( $>99.9 \%)(v / v)$ was purchased from Sinopharm Chemical Reagent Co. (Beijing, China). Concentrations of 2, $5,10,20$ and $50 \%$ ethanol (v/v) were prepared with $>99.9 \%$ ethanol and sterile water. Concentrations of 2, 5, 10, 20 and $50 \%$ ethanol-DDP were freshly prepared with $8 \mathrm{mg} / \mathrm{kg}$ DDP (Qilu Pharmaceutical Co., Ltd.) dissolved in a corresponding concentration of ethanol solution of $150 \mu \mathrm{l}$.

Tumor growth xenograft study. A total of 180 inbred male Balb/C nude mice, aged 6 weeks, were obtained from the Institute of the Chinese Association for Laboratory Animal Sciences (Beijing, China). A549/DDP lung adenocarcinoma cells $\left(5 \times 10^{6}\right)$ were subcutaneously inoculated in the upper left flank on day 1 . When the tumor diameters had exceeded $5 \mathrm{~mm}$, the mice were divided into the control group, the 2, 5 , 10,20 and $50 \%$ ethanol groups, the DDP group and the 2, $5,10,20$ and $50 \%$ ethanol + DDP groups ( $\mathrm{n}=15$ per group). The mice in each group were treated with intratumoral injections of $150 \mu \mathrm{l}$ sterile water (control); 2, 5, 10, 20 or $50 \%$ ethanol; $8 \mathrm{mg} / \mathrm{kg}$ DDP in sterile water; or $2,5,10,20$ or $50 \%$ ethanol $+8 \mathrm{mg} / \mathrm{kg}$ DDP, accordingly, twice a week for 4 weeks. Tumor volumes were measured with calipers twice a week and calculated using the following equation: (width ${ }^{2} \mathrm{x}$ length)/2 (5). The survival of mice in each group was observed and recorded. To minimize the suffering of the animals, cleaning practices were monitored on a regular basis to ensure effective hygiene and sterile sanitation. To avoid unnecessary harm, the agents were injected gently. The condition of the animals was monitored daily for evidence of illness. Pain reaction associated with intratumoral injection was also observed. Mice showing severe distress, including infection, ulceration, cachexia, inability to ambulate and moribund were euthanized by cervical dislocation in accordance with animal care protocols. All the mice were euthanized after 180 days of observation.

ICH staining of CD34 in tumor tissues. The paraffin-embedded tissues were cut into $4-\mu \mathrm{m}$ sections and heated at $60^{\circ} \mathrm{C}$ for $60 \mathrm{~min}$. The sections were deparaffinized with xylene and rehydrated, then treated with $3 \%$ hydrogen peroxide in methanol, followed by incubation with normal serum to block non-specific binding. The slides were incubated with anti-mouse CD34 antibody (Abcam, Cambridge, UK) at $4^{\circ} \mathrm{C}$ overnight. After washing, the tissue sections were treated with anti-rat secondary antibody (Zhongshan Golden Bridge Biotechnology Co., Ltd., Beijing, China), followed by further incubation with horseradish peroxidase-labelled polymer (Dako, Carpinteria, CA, USA) for $20 \mathrm{~min}$. Following staining with diaminobenzidine (Sigma-Aldrich, Munich, Germany), the sections were counterstained with hematoxylin (Sigma-Aldrich). The known positive tissues were used as positive controls and the primary antibody was omitted for negative controls. The staining of targeted proteins was visualized under an Olympus microscope (Olympus, Tokyo, Japan).

The CD34 antibody-stained slides were reviewed and scored independently by two observers. MVD was detected using immunostaining, as previously described by Weidner (17). The mean score of 5 fields was considered to be the level of MVD for each sample.

Statistical analysis. Data are expressed as means \pm standard deviation except in the analysis of survival data, which are expressed as means \pm standard error of the mean. Paired and unpaired Student's t-tests were used to analyze two groups of paired or unpaired data, respectively. Repeated measured analysis of variance was used for the comparison of multiple groups. Survival analysis was performed according to the Kaplan-Meier method and the log-rank (Mantel-Cox) test using SPSS statistical software version 19.0 (IBM, Armonk, NY, USA). Mice that remained alive at the end of the study were censored. $\mathrm{P}<0.05$ was considered to indicate a statistically significant difference. 
A

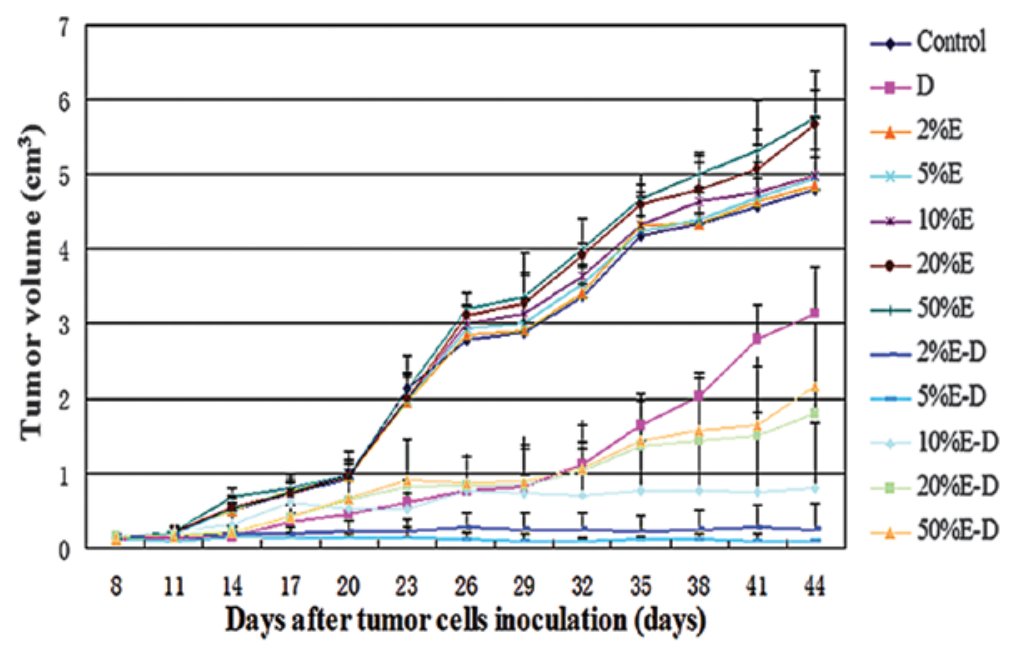

B

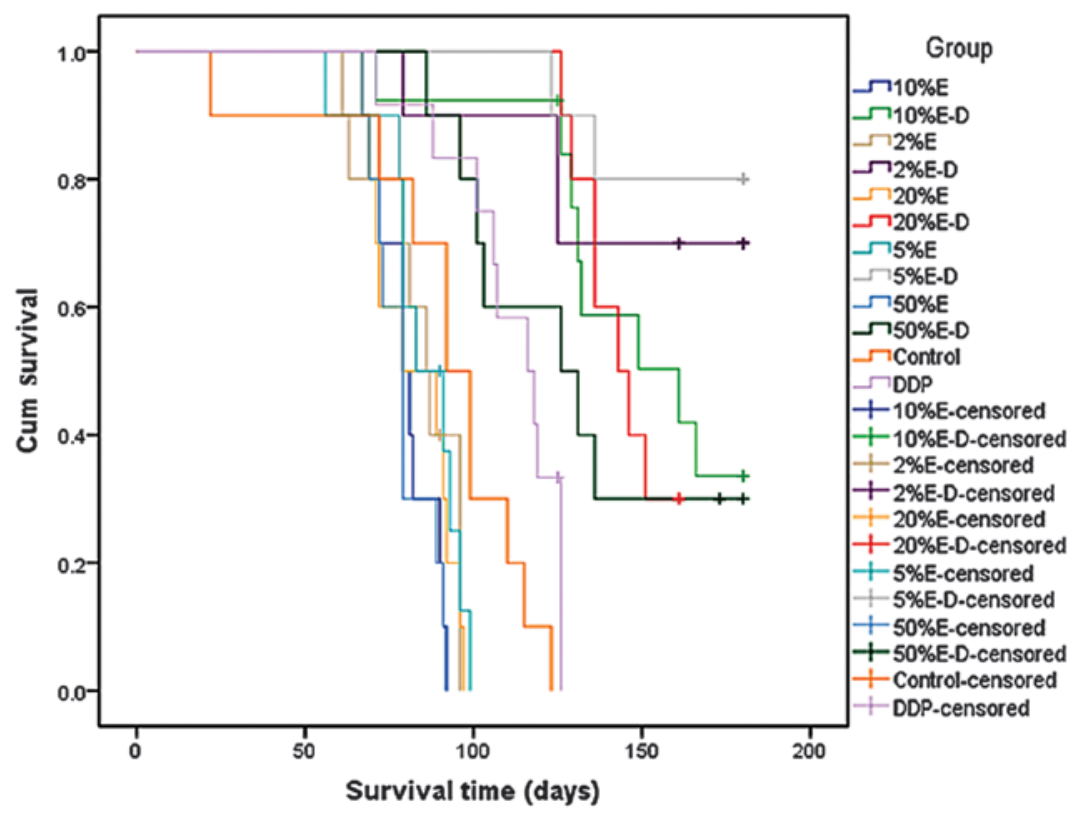

Figure 1. Changes in tumor size and survival in DDP-resistant lung tumor-bearing Balb/C mice following treatment with DDP in various concentrations of ethanol. (A) Changes in tumor size following various treatments. After 4 weeks of treatment, the mean tumor volume in mice treated with $5 \%$ ethanol-DDP $\left(0.11 \pm 0.06 \mathrm{~cm}^{3}\right)$ was statistically lower compared to that in mice treated with $2,10,20$ and $50 \%$ ethanol-DDP $(0.22 \pm 0.08,0.78 \pm 0.36,1.36 \pm 0.53$ and $1.43 \pm 0.71 \mathrm{~cm}^{3}$, respectively; $\mathrm{P}<0.01$ ). Treatment with 5\% ethanol-DDP was the most effective in reducing tumor volumes in DDP-resistant tumor-bearing mice. (B) Survival of A549/DDP tumor-bearing mice following various treatments. Treatment with 5\% ethanol-DDP achieved the longest estimated mean survival among all the treatment groups. The estimated mean survival time in 5\% ethanol-DDP-treated mice (169.9 \pm 6.5 days) was significantly longer compared to that in mice treated with 10, 20 and 50\% ethanol-DDP $(149.3 \pm 8.8,145.0 \pm 4.0$ and $131.9 \pm 11.0$ days, respectively; $\mathrm{P}<0.05)$. Although there were no significant differences between 2 and 5\% ethanol-DDP-treated mice, the estimated mean survival time in the 5\% ethanol-DDP mice tended to be longer compared to that in the $2 \%$ ethanol-DDP-treated mice (169.9 \pm 6.5 vs. $158.9 \pm 10.9$ days; $\mathrm{P}>0.05)$. E, ethanol; D/DDP, cisplatin; cum, cumulative.

\section{Results}

Tumor size changes in different groups of DDP-resistant lung tumor-bearing mice. After 4 weeks of treatment, the mean tumor volume in the $5 \%$ ethanol-DDP group $\left(0.11 \pm 0.06 \mathrm{~cm}^{3}\right)$ was statistically lower compared to that in the $2,10,20$ or $50 \%$ ethanol-DDP groups $\left(0.22 \pm 0.08 \mathrm{~cm}^{3}, 0.78 \pm 0.36 \mathrm{~cm}^{3}\right.$, $1.36 \pm 0.53 \mathrm{~cm}^{3}$ and $1.43 \pm 0.71 \mathrm{~cm}^{3}$, respectively; $\left.\mathrm{P}<0.01\right)$ (Fig. 1A). Administration of 5\% ethanol-DDP to A549/DDP tumor-bearing mice resulted in complete tumor regression in 3 out of 10 mice (30\%) and tumor growth arrest in the remaining 7 mice $(70 \%)$ after 4 weeks of treatment. By contrast, only 1 out of 10 mice (10\%) treated with $2 \%$ ethanol-DDP and no mice in the other groups achieved complete tumor regression.
Compared to the tumor size in control mice $\left(3.68 \pm 0.48 \mathrm{~cm}^{3}\right)$, treatment with $2,5,10,20$ or $50 \%$ ethanol alone was shown to stimulate tumor growth $\left(4.31 \pm 0.12 \mathrm{~cm}^{3}, \mathrm{P}<0.01 ; 4.23 \pm 0.63 \mathrm{~cm}^{3}\right.$, $\mathrm{P}<0.05 ; 4.32 \pm 0.63 \mathrm{~cm}^{3}, \mathrm{P}<0.01 ; 4.59 \pm 0.36 \mathrm{~cm}^{3}, \mathrm{P}<0.01$; and $4.67 \pm 0.26 \mathrm{~cm}^{3}, \mathrm{P}<0.01$, respectively) (Fig. 1A).

Survival of tumor-bearing nude mice in different groups. Treatment with 5\% ethanol-DDP achieved the longest survival among all the investigated treatment groups. After 180 days of observation, $80 \%$ ( 8 out of 10 ) of the $5 \%$ ethanol-DDP-treated mice remained alive, with two deaths from non-tumor-related reasons; all 10 control mice died prior to day 123 .

The mean survival of the $5 \%$ ethanol-DDP-treated group (169.9 \pm 6.5 days) was found to be significantly longer compared 


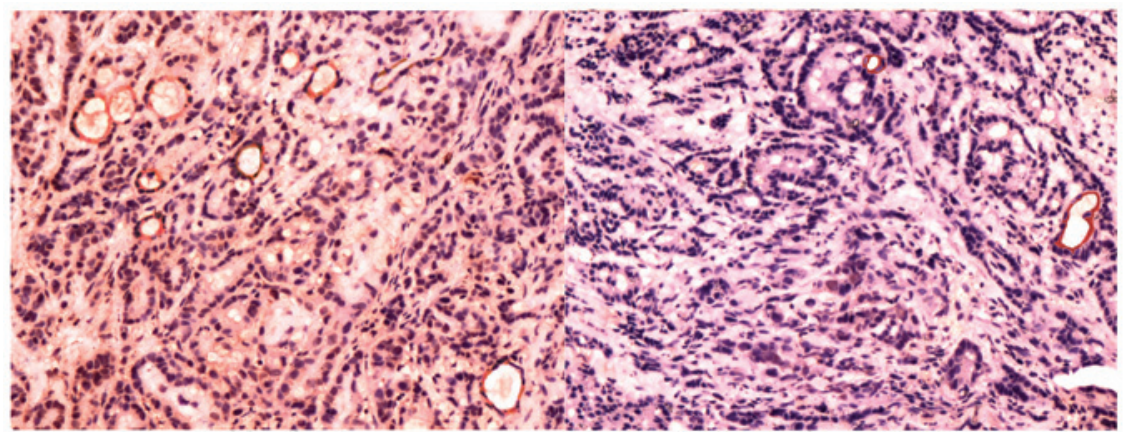

\section{Control}

$10 \%$ ethanol-DDP

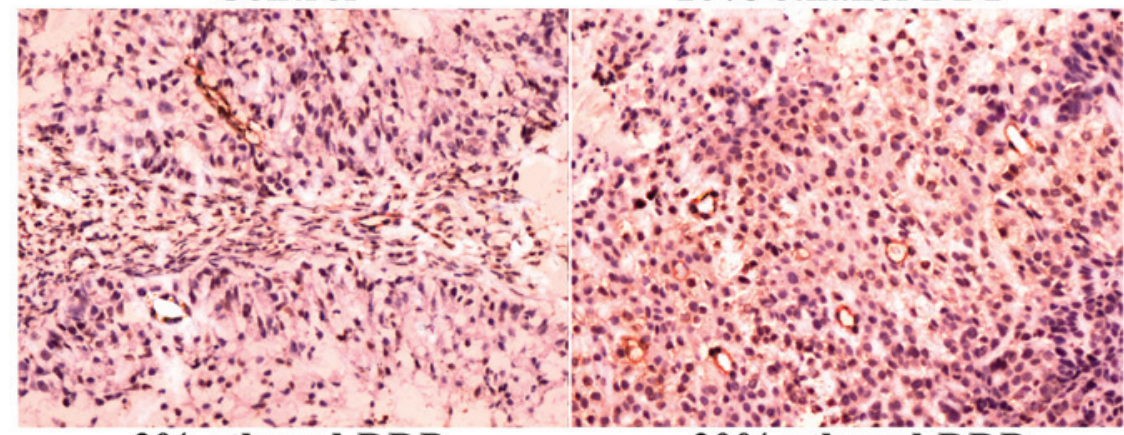

$2 \%$ ethanol-DDP $20 \%$ ethanol-DDP

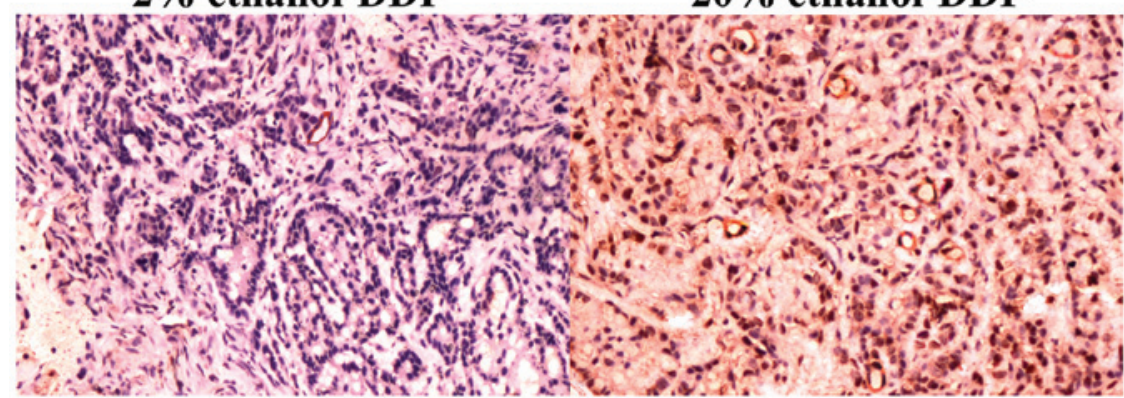
$5 \%$ ethanol-DDP

$50 \%$ ethanol-DDP

Figure 2. Immunohistochemistry analysis of microvessel density (MVD) in tumor tissues. MVD staining of tumor tissues in control (upper left panel), 2\% (middle left panel), 5\% (bottom left panel), 10\% (upper right panel), 20\% (middle right panel) and 50\% ethanol-DDP-treated mice (bottom right panel) Compared to the MVD of 30.2 \pm 3.5 in control tumor tissues, treatment with 2,5 and 10\% ethanol-DDP significantly decreased MVD in tumor tissues (21.3 \pm 3.6 , $\mathrm{P}<0.01 ; 15.3 \pm 3.1, \mathrm{P}<0.01$; and $23.6 \pm 3.1, \mathrm{P}<0.05$, respectively). Among these, $5 \%$ ethanol-DDP produced the most significant MVD reduction compared to that in the control samples. The MVD in the tumor tissues of mice treated with 20 and 50\% ethanol-DDP was not significantly altered compared to the MVD of the control tissues (26.5 \pm 5.3 and $33.3 \pm 5.6$; P $>0.05$, respectively). DDP, cisplatin.

to that of the 10,20 and $50 \%$ ethanol-DDP, DDP, control and 2, 510,20 and $50 \%$ ethanol alone groups $(149.3 \pm 8.8$ days, $\mathrm{P}<0.05 ; 145.0 \pm 4.0$ days, $\mathrm{P}<0.05 ; 131.9 \pm 11.0$ days, $\mathrm{P}<0.05$ $110.8 \pm 5.1$ days, $\mathrm{P}<0.01 ; 90.6 \pm 9.0$ days, $\mathrm{P}<0.01 ; 84.1 \pm 4.3$ days, $\mathrm{P}<0.01 ; 84.9 \pm 4.1$ days, $\mathrm{P}<0.01 ; 80.2 \pm 2.8$ days, $\mathrm{P}<0.01$; $82.3 \pm 3.8$ days for $20 \%, \mathrm{P}<0.01$; and $79.0 \pm 2.9$ days, $\mathrm{P}<0.01$, respectively). Although there were no significant differences between 2 and $5 \%$ ethanol-DDP-treated mice, the estimated mean survival time of $5 \%$ ethanol-DDP-treated mice tended to be longer compared to that of the $2 \%$ ethanol-DDP-treated mice (169.9 \pm 6.5 vs. $158.9 \pm 10.9$ days, respectively; $\mathrm{P}>0.05$ ) (Fig. 1B).

Effects of various combinations of ethanol-DDP on tumor angiogenesis by IHC analysis of MVD. Compared to the MVD of 30.2 \pm 3.5 in the control group, treatment with 2, 5 and $10 \%$ ethanol-DDP significantly decreased MVD in the tumor tissues $(21.3 \pm 3.6, \mathrm{P}<0.01 ; 15.3 \pm 3.1, \mathrm{P}<0.01$; and $23.6 \pm 3.1, \mathrm{P}<0.05$, respectively). Among these combinations,
$5 \%$ ethanol-DDP produced the most significant MVD reduction compared to that in the control group (Fig. 2). MVD in tumor tissues treated with 20 and 50\% ethanol-DDP was not significantly altered compared to the MVD of the control group $(26.5 \pm 5.3$ and $33.3 \pm 5.6$, respectively; $\mathrm{P}>0.05)$. Compared to a MVD of 30.2 \pm 3.5 in the control group, various concentrations of ethanol alone increased MVD $(42.2 \pm 5.8$ for $2 \%, 50.7 \pm 5.3$ for $5 \%, 57.4 \pm 6.1$ for $10 \%, 61.7 \pm 7.3$ for $20 \%$ and $65.3 \pm 6.1$ for $50 \%$ ethanol; $\mathrm{P}<0.01)$. In addition to the stimulation of tumor growth, ethanol at higher concentrations, such as 20 and $50 \%$, also caused local injection pain.

\section{Discussion}

Our study demonstrated that treatment with 5\% ethanol-DDP achieved the highest efficiency among all the investigated ethanol-DDP combinations in reducing tumor volume and prolonging survival. The results of the MVD analysis suggested that the potent inhibition of tumor angiogenesis by 
$5 \%$ ethanol-DDP may be one of the key mechanisms underlying its superior efficiency.

Treatment with 5\% ethanol-DDP achieved the most significant tumor growth inhibition compared to 2, 5, 10, 20 and $50 \%$ ethanol-DDP. Treatment with 5\% ethanol-DDP also induced complete tumor regression in 3 out of 10 mice $(30 \%)$ and tumor growth arrest in the remaining 7 mice (70\%) after 4 weeks of treatment. By contrast, only 1 of the 10 mice $(10 \%)$ treated with $2 \%$ ethanol-DDP and no mice in the other groups achieved complete tumor regression.

In addition, 5\% ethanol-DDP treatment produced the longest survival among all groups of mice. After 180 days of observation, $80 \%$ (8 out of 10 ) of the $5 \%$ ethanol-DDP-treated mice remained alive, with two deaths from non-tumor-related reasons.

Mechanistically, 5\% ethanol-DDP exhibited the most significant tumor angiogenesis inhibition among all the investigated ethanol-DDP combinations, whereas various concentrations of ethanol alone were shown to increase tumor angiogenesis. Our results suggested that DDP inhibited tumor angiogenesis most potently in 5\% ethanol and this may be one of the mechanisms underlying its superior efficiency.

Previous studies by Pietronigro et al (18) and Jenkinson et al (19) reported that intratumoral injection of the chemotherapeutic agent carmustine dissolved in $100 \%$ ethanol, achieved a $40 \%$ cure rate in rats bearing intracranial T9 tumors and $72 \%$ stable disease in patients with recurrent malignant glioma. However, our results demonstrated that DDP, when dissolved in high concentrations of ethanol, such as $50 \%$ ethanol, exhibited minimal tumor inhibition with obvious normal tissue damage compared to DDP dissolved in lower ethanol concentrations. We also observed that higher concentrations of ethanol significantly promoted tumor growth, which was associated with increased tumor angiogenesis. These results were consistent with those of previous studies by Tan et al (13) and Forsyth et al (14), suggesting that ethanol alone stimulates angiogenesis and promotes tumor growth. A possible explanation for the differences between our results and those of previous studies may be that the glioma tumors in the rats in those studies were smaller compared to the tumors in our study. Smaller tumors may be easier to diffuse and sufficiently suffuse by $100 \%$ ethanol, resulting in complete tumor necrosis. By contrast, the tumors in our studies were difficult to evenly diffuse due to their large size and required higher concentrations of ethanol, leaving some tumor cells unharmed and activating tumor angiogenesis.

In our previous xenograft study, we demonstrated that 5\% ethanol-DDP injected intratumorally was able to eradicate DDP-resistant lung tumors and prolong survival by eliminating lung CSCs and non-CSC cancer cells (15). However, whether there was an optimal ethanol concentration in which DDP achieved a higher efficacy compared to that of 5\% ethanol-DDP had not been determined. The potential mechanisms underlying the efficiency of DDP in different concentrations of ethanol had not been fully elucidated. The present study established that 5\% ethanol-DDP was the optimal combination compared to that of DDP with 2, 5, 10, 20 and $50 \%$ ethanol in controlling DDP-resistant lung tumors. We also found that $5 \%$ ethanol-DDP inhibited tumor angiogenesis most significantly compared to other combinations and may be one of the mechanisms underlying its superior efficiency.

This study confirmed 5\% ethanol-DDP as the optimal combination for the treatment of DDP-resistant lung tumors among the combinations of DDP with 2, 5, 10, 20 and $50 \%$ ethanol. Ethanol and DDP have been used safely in the clinical setting over several decades; however, the application of this combination to improve the prognosis of DDP-resistant lung cancer warrants further investigation.

In conclusion, our results demonstrated that $5 \%$ ethanol-DDP achieved the most potent tumor growth inhibition and prolongation of survival compared to the other investigated ethanol-DDP combinations, providing an effective method to improve the survival of patients with DDP-resistant lung cancer. However, further clinical studies are required to clearly determine the efficacy and safety of this novel approach.

\section{Acknowledgements}

The authors would like to thank Dr Li Li (Tuberculosis Research Institute, 309 PLA Hospital) for his assistance and valuable advice in the animal assay. This study was supported by the Scientific Research Foundation for the Returned Overseas Chinese Scholars, China State Education Ministry (grant no. 2007-1108).

\section{References}

1. Jemal A, Bray F, Center MM, et al: Global cancer statistics. CA Cancer J Clin 61: 69-90, 2011.

2. Rossi A, Di Maio M, Chiodini P, et al: Carboplatin- or cisplatin-based chemotherapy in first-line treatment of small-cell lung cancer: the COCIS meta-analysis of individual patient data. J Clin Oncol 30: 1692-1698, 2012.

3. Ardizzoni A, Boni L, Tiseo M, et al; CISCA (CISplatin versus CArboplatin) Meta-analysis Group: Cisplatin- versus carboplatin-based chemotherapy in first-line treatment of advanced non-small-cell lung cancer: an individual patient data meta-analysis. J Natl Cancer Inst 99: 847-857, 2007.

4. Eramo A, Haas TL and De Maria R: Lung cancer stem cells: tools and targets to fight lung cancer. Oncogene 29: 4625-4635, 2010.

5. Niu Q, Wang W, Li Y, Ruden DM, Wang F, Li Y, Wang F, Song J and Zheng K: Low molecular weight heparin ablates lung cancer cisplatin-resistance by inducing proteasome-mediated ABCG2 protein degradation. PLoS One 7: e41035, 2012.

6. Dean M, Fojo $\mathrm{T}$ and Bates S: Tumour stem cells and drug resistance. Nat Rev Cancer 5: 275-284, 2005.

7. Singh A, Wu H, Zhang P, Happel C, Ma J and Biswal S: Expression of ABCG2 (BCRP) is regulated by Nrf2 in cancer cells that confers side population and chemoresistance phenotype. Mol Cancer Ther 9: 2365-2376, 2010.

8. Zhang M, Mathur A, Zhang Y, et al: Mithramycin represses basal and cigarette smoke-induced expression of ABCG2 and inhibits stem cell signaling in lung and esophageal cancer cells. Cancer Res 72: 4178-4192, 2012.

9. Kim ES, Lee JJ, He G, Chow CW, Fujimoto J, Kalhor N, Swisher SG, Wistuba II, Stewart DJ and Siddik ZH: Tissue platinum concentration and tumor response in non-small-cell lung cancer. J Clin Oncol 30: 3345-3352, 2012.

10. Tang CH, Parham C, Shocron E, McMahon G and Patel N: Picoplatin overcomes resistance to cell toxicity in small-cell lung cancer cells previously treated with cisplatin and carboplatin. Cancer Chemother Pharmacol 67: 1389-1400, 2011.

11. Livraghi T, Festi D, Monti F, Salmi A and Vettori C: US-guided percutaneous alcohol injection of small hepatic and abdominal tumors. Radiology 161: 309-312, 1986.

12. Kuang M, Lu MD, Xie XY, Xu HX, Xu ZF, Liu GJ, Yin XY, Huang JF and Lencioni R: Ethanol ablation of hepatocellular carcinoma up to $5.0 \mathrm{~cm}$ by using a multipronged injection needle with high-dose strategy. Radiology 253: 552-561, 2009. 
13. Tan W, Bailey AP, Shparago M, Busby B, Covington J, Johnson JW, Young E and Gu JW: Chronic alcohol consumption stimulates VEGF expression, tumor angiogenesis and progression of melanoma in mice. Cancer Biol Ther 6: 1211-1217, 2007.

14. Forsyth CB, Tang Y, Shaikh M, Zhang L and Keshavarzian A: Alcohol stimulates activation of Snail, epidermal growth factor receptor signaling, and biomarkers of epithelial-mesenchymal transition in colon and breast cancer cells. Alcohol Clin Exp Res 34: 19-31, 2010

15. Niu Q, Wang W, Li Y, Ruden DM, Li Q and Wang F: Cisplatin in 5\% ethanol eradicates cisplatin-resistant lung tumor by killing lung cancer side population (SP) cells and non-SP cells. Front Genet 4: 163, 2013.

16. Hamstra DA, Moffat BA, Hall DE, Young JM, Desmond TJ, Carter J, Pietronigro D, Frey KA, Rehemtulla A and Ross BD: Intratumoral injection of BCNU in ethanol (DTI-015) results in enhanced delivery to tumor - a pharmacokinetic study. J Neurooncol 73: 225-238, 2005
17. Weidner N: Current pathologic methods for measuring intratumoral microvessel density within breast carcinoma and other solid tumors. Breast Cancer Res Treat 36: 169-180, 1995.

18. Pietronigro D, Drnovsky F, Cravioto $\mathrm{H}$ and Ransohoff $\mathrm{J}$ DTI-015 produces cures in T9 gliosarcoma. Neoplasia 5: $17-22,2003$

19. Jenkinson MD, Smith TS, Haylock B, Husband D, Shenoy A, Vinjamuri S, Walker C, Pietronigro D and Warnke PC: Phase II trial of intratumoral $\mathrm{BCNU}$ injection and radiotherapy on untreated adult malignant glioma. J Neurooncol 99: 103-113, 2010. 